\title{
Diagnóstico de enfermagem em urgência e emergência
}

\author{
Nursing diagnosis in urgency and emergency \\ Diagnóstico de enfermería en urgencias y emergencias
}

Recebido: 18/10/2021 | Revisado: 25/10/2021 | Aceito: 26/10/2021 | Publicado: 29/10/2021

\author{
Israel Aparecido dos Santos \\ ORCID: https://orcid.org/0000-0001-9933-5991 \\ Faculdade Cristo Rei, Brasil \\ E-mail: picicro@gmail.com \\ Thiarles Cristian Aparecido \\ ORCID: https://orcid.org/0000-0003-0029-3758 \\ Faculdade Cristo Rei, Brasil \\ E-mail: thiarles@faccrei.edu.br
}

\begin{abstract}
Resumo
O presente artigo relata sobre o diagnóstico de enfermagem em urgência e emergência, sendo de extrema importância $\mathrm{o}$ atendimento hospitalar levando em consideração todas as condições momentâneas relativas ao estado geral do paciente, o profissional de enfermagem executa sua atividade com agilidade e competência no primeiro atendimento. A pesquisa tem como objetivos relatar o papel do profissional de saúde e suas importâncias nos diagnósticos de urgência e emergências e no atendimento hospitalar e evidenciar a prioridade dos desenvolvimentos dos diagnósticos coletados pelos profissionais de urgência e emergência e ajudar os profissionais do pronto socorro a ter uma agilidade maior para os cuidados do paciente. O presente artigo adota como metodologia os recursos de revisão bibliográfica, sobre o aspecto de temas históricos, empregas sobre o estudo de referências baseado com documentação indireta, com pesquisas de artigos científicos, pesquisa documental e pesquisas bibliográficas, realizado em quatro etapas, identificação do problema, buscar de literatura, avaliação e análise de dados, e por final a síntese da revisão bibliográfica. Como resultados e Discussão este estudo atua na importância do diagnóstico de enfermagem em urgência e emergência, sendo considerada um grande desafio, pois a cada dia essa área de saúde há superlotação assim para manter a qualidade da assistência é muito complicada. Concluindo sobre a importância dos diagnósticos e a relevância das coletas de dados pelos profissionais de saúde, podemos ajudar a salvar muitas vidas que seria perdida pela falta dos diagnósticos deixados de ser coletados no ato do atendimento.
\end{abstract}

Palavras-chave: Diagnóstico; Urgência; Emergência.

\begin{abstract}
This article reports on the nursing diagnosis in urgency and emergency, being of extreme importance the hospital care taking into account all the momentary conditions related to the general state of the patient, the nursing professional performs his activity with agility and competence in the first visit. The research aims to report the role of the health professional and its importance in urgent and emergency diagnoses and in hospital care and highlight the priority of the developments of diagnoses collected by urgent and emergency professionals and help emergency room professionals to have a greater agility for patient care. This article adopts as a methodology the resources of bibliographic review, on the aspect of historical themes, employs on the study of references based on indirect documentation, with research of scientific articles, documentary research and bibliographic research, carried out in four stages, identification of the problem, literature search, data evaluation and analysis, and finally the synthesis of the bibliographic review. As results and Discussion this study acts on the importance of nursing diagnosis in urgency and emergency, being considered a great challenge, because every day this area of health there is overcrowding, so to maintain the quality of care is very complicated. Concluding about the importance of diagnoses and the relevance of data collection by health professionals, we can help to save many lives that would be lost due to the lack of diagnoses not collected during the service.
\end{abstract}

Keywords: Diagnossi; Urgency; Emergency.

\section{Resumen}

Este artículo informa sobre el diagnóstico de enfermería en urgencia y emergencia, siendo la atención hospitalaria de suma importancia, teniendo en cuenta todas las condiciones momentáneas relacionadas con el estado general del paciente, el profesional de enfermería realiza su actividad con agilidad y competencia en la primera asistencia. La investigación tiene como objetivo dar a conocer el papel del profesional de la salud y su importancia en los diagnósticos de urgencia y emergencia y en la atención hospitalaria y resaltar la prioridad de los desarrollos de diagnósticos recogidos por los profesionales de urgencias y emergencias y ayudar a los profesionales de urgencias a 
tener una mayor agilidad para el paciente. cuidado. Este artículo adopta como metodología los recursos de revisión bibliográfica, en el aspecto de temática histórica, emplea en el estudio de referencias a partir de documentación indirecta, con investigación de artículos científicos, investigación documental e investigación bibliográfica, realizada en cuatro etapas, identificación de el problema, búsqueda bibliográfica, evaluación y análisis de datos, y finalmente la síntesis de la revisión bibliográfica. Como resultados y Discusión este estudio actúa sobre la importancia del diagnóstico de enfermería en urgencia y emergencia, siendo considerado un gran desafío, pues todos los días esta área de la salud se encuentra masificada, por lo que mantener la calidad de la atención es muy complicado. Concluyendo sobre la importancia de los diagnósticos y la relevancia de la recolección de datos por parte de los profesionales de la salud, podemos ayudar a salvar muchas vidas que se perderían por la falta de diagnósticos no recolectados durante el servicio.

Palabras clave: Diagnóstico; Urgencia; Emergencia.

\section{Introduçãa}

A relevância deste estudo aponta sobre o enfermeiro no ato do atendimento em um ambiente hospitalar de urgência e emergência, devendo avaliar a real situação em que o paciente se encontra no exato momento. As ações de enfermagem em urgência e emergência devem ocorrer de forma ágil, eficaz e habilidosa.

A urgência e emergência são normalmente reconhecidas como aquelas que se encontram à pronta atenção médica e necessária. Os diagnósticos são a base dos levantamentos de dados coletado pelo profissional de saúde dentro de um quadro de atendimento aos pacientes. Cada paciente recebe um tipo de cuidado priorizado (Rocha, 2012).

$\mathrm{Na}$ hipótese do enfermeiro com a capacitação para atuar em ambientes de urgência e emergência hospitalares, com habilidades em teorias e práticas, à qualquer situação e agravo para manter o bem-estar do paciente. O presente estudo tem como objetivo principal relatar o papel do profissional de saúde e suas importâncias nos diagnósticos de urgência e emergências e no atendimento hospitalar. E como objetivos específicos desenvolver pesquisa teórica a respeito dos assuntos urgência e emergências no atendimento hospitalar, demostrar a importância do papel do enfermeiro em diferentes ambientes de saúde, especialmente no atendimento hospitalar e apresentar os dados coletados, em forma de artigo científico.

A procura por atendimento de emergência aumenta a cada dia, devido à complexidade e gravidades dos pacientes e a super lotação nesse ambiente, faze com que os profissionais de saúde não consigam atender de maneira eficiente e eficaz a fim de promover o diagnostico correto, os acontecimentos de urgência e emergência são normalmente reconhecidos como situações que acometem a atenção médica onde se faz necessária o atendimento rápido. Os diagnósticos são à base dos levantamentos de dados prescritos pelo enfermeiro dentro de uma situação de atendimento ao paciente em um pronto socorro. (Dutra, 2014).

Algumas dificuldades enfrentadas pela equipe de enfermagem a área de emergência, como a falta de funcionários, falta de respaldo institucional e profissional, carga de trabalho elevada, espaço físico inadequado e conflito com usurários e familiares contribuem para insegurança do profissional de saúde, podendo não ter o seu atendimento livre de riscos, sendo possível causar algum impacto à saúde do paciente. (Sallum, 2012).

De acordo com a Resolução COFFEN, no. 358 de 15 de outubro de 2009, o Conselho Federal de Enfermagem ressalva as necessidades de implementação do processo de enfermagem nos serviços de saúde, uma atividade privativa dos profissionais de saúde, para que tenham um cuidado de enfermagem seguro e humanizado de acordo com as exigências do paciente. (COFEN, 2009).

O diagnóstico de enfermagem recomendado pela North American Nursing Diagnosis Association (NANDA) fornece uma linguagem padronizada, utilizando como produtos do raciocínio e julgamento clínico sobre as respostas humanas aos problemas de saúde e processos vitais essenciais ao paciente.

Os cuidados de acordo com o diagnóstico de enfermagem se fazem importante porque é a concretização de um método para nortear o cuidado ao paciente na sua integralidade. De acordo com situação do paciente se faz necessária à 
assistência de enfermagem com um método de trabalho indispensável para as ações organizadas e o atendimento necessário. (Passo, 2011).

\subsection{Diagnóstico de enfermagem}

O diagnóstico de enfermagem real relata as respostas humanas e condições de saúde vitais do indivíduo, família ou comunidade. No diagnóstico de enfermagem de promoção de saúde o julgamento clínico de motivação e de desejo do indivíduo, família ou comunidade, tem como intuito aumentar o a qualidade de vida e potencial da saúde humana. O diagnóstico de enfermagem de risco descreve respostas humanas que desenvolvem um indivíduo família ou comunidade vulneráveis e o diagnóstico de enfermagem de bem-estar. (Garcia, 2019)

Após essa avaliação o profissional de saúde tem como avaliar a necessidades do paciente, com o processo sistemático e continuo relacionado com técnicas semiológicas dentro da propedêutica para obter informações sobre o paciente e sua família esses dados são coletados através de observação e o que o paciente relata. (Soares, 2015).

No atendimento inicial no setor de urgência e emergência realizado pelo profissional de saúde há classificação de risco de acordo com o protocolo de Manchester. O risco de agravamento por cores: onde para a cor vermelha há prioridade zero, para a laranja existe tempo limite de espera até 10 minutos, no amarelo, tempo limite de espera até 60 minutos, o verde possui tempo limite de espera até 120 minutos e azul, tempo limite de espera até 240 minutos. Assim os pacientes são direcionados ao atendimento médico de acordo com suas necessidades. (COFEN, 2012).

O procedimento de diagnostico no setor de emergência exige uma intervenção imediata, pois são situações de caráter mais imediatos, vez que pode haver risco de morte, como por exemplo: cortes profundos, acidentes de origem elétrica, picadas ou mordidas de animais peçonhentos, queimaduras, afogamentos, hemorragias, infarto do miocárdio, dificuldade respiratória, derrames, inconsciência, desmaio, intoxicação por alimentos ou remédios, sangue no vômito, urina ou tosse, graves reações alérgicas, febre alta permanente, convulsões, agressões físicas e, acidentes automotivos e automobilísticos e quedas. (Garcia, 2019).

\subsection{Urgência e emergência}

A urgência e emergência são palavras usadas em ambientes da medicina, termos que podem ser confundidos, como por exemplo: emergência é algo que não pode ser adiado, precisa ser atendido no ato. Urgência ocorre quando existe uma situação crítica dentro dos parâmetros da medicina, se não atendido corretamente pode se tornar em emergência. (Oliveira, 2015)

Assim, urgência e emergência são definidos como processos diferentes. São de extremidades opostas, um lado como ausência de risco de vida e outro como a existência de risco máximo de vida onde ambos contribuem juntos para salvar vida (Oliveira, 2015). Dessa forma, é necessário que os casos de urgência e emergência sejam realizados por profissionais treinados e capacitados para prestar atendimento humanizado e integral aos usuários de forma rápida e eficaz (Costa, et al., 2020)

De acordo com o ministério de saúde, a emergência é vista como condições de risco a saúde o que pode levar ao sofrimento intenso e até a morte, assim deve ter o tratamento médico imediato - a urgência é uma ocorrência imprevista de riscos à saúde podendo ter risco ou não a vida humana, onde o paciente necessita de assistência médica imediata (Brasil, 2014).

A área de urgência e emergência é muito importante para a assistência à saúde, porque a cada dia cresce mais os serviços nesta área, devido eventos relacionados a acidentes, violência urbana, doenças agudas e crônicas. Ó atendimento à população vem se transformando numa das mais problemáticas áreas do sistema de saúde. O Sistema Único de Saúde (SUS) tem com obrigação estar organizando a assistência desde a unidade de atenção primaria até as terciárias, estendendo aos cuidados pós-hospitalar na convalescência, recuperação e reabilitação (Brasil, 2013). 
De acordo com a política nacional de atenção as urgências, o atendimento de urgências e emergências tem como objetivo atender pacientes em estado graves e não tão urgentes, que logo após serão encaminhados às redes especializadas de atenção à saúde. Assim, tem-se que o setor considerado como pronto atendimento é definido como unidade prestadora de atendimento à saúde, com atendimento 24 horas por dia, onde são realizados os primeiros atendimentos e logo em seguida encaminha a unidade especializada (Brasil, 2013).

\subsection{A importância do profissional de enfermagem em urgência e emergência}

O papel do profissional de enfermagem em emergência e urgência tem como prioridade analisar o paciente, fazer exame físico, executar o tratamento, aconselhar e ensinar o paciente os cuidados com sua saúde. A função do profissional de enfermagem no setor de urgência e emergência hospitalar é o aconselhamento ao paciente e sua família, realizar os procedimentos necessários ao nível de risco que o paciente se encontra. Essa avalição ao paciente é de forma primária, dar um suporte básico e avançado de vida, fazer um atendimento à vítima de trauma, dar assistência ao infarto agudo do miocárdio e o acidente vascular encefálico e também dar assistência as emergências obstétricas. No setor de atendimento de urgência e emergência o profissional de saúde é o protagonista porque está sempre realizando, gerenciando e cuidando do paciente ao mesmo tempo, ele tem autonomia para decisões com capacidade de avaliar e cuidar da assistência integral e sem danos ao paciente (Munhos, 2016).

A atuação do profissional de saúde na área de urgência e emergência começa na classificação de risco, reposição do carro de emergência, supervisão e capacitação de sua equipe, avaliação da assistência prestada e realização da sistematização a assistência de enfermagem (Filho, 2016).

O profissional de enfermagem desenvolve um atendimento ao paciente rápido e eficaz, muitas vezes começa esse atendimento nas unidades móveis, já com os procedimentos, mantendo a sobrevivência do paciente até chegar à rede hospitalar (Rocha, 2012).

Sua atuação pode ser desenvolvida em postos de saúde, hospitais e demais serviços de saúde, esse profissional deve tomar sempre atitudes que leve em conta seu conhecimento teórico e prático. $\mathrm{Na}$ área de urgência e emergência sua atuação é bem ampla pode avaliar a assistência prestada, realizar e classificar os riscos, supervisionar e capacitar. (Oliveira, 2015)

A importância do profissional de saúde no setor de urgência e emergência está ligada na sua atuação, pois muitas vezes se depara com situações muito delicadas, muitas vezes com paciente em estado muito grave, atentar em ter um atendimento humanizado além de oferecer ao paciente uma assistência segura. (Rocha, 2012)

\section{Materiais e Métodos}

O presente artigo adota como metodologia os recursos de revisão bibliográfica integrativa, sobre o aspecto de temas históricos, empregas sobre o estudo de referências baseado com documentação indireta, com pesquisas de artigos científicos, pesquisa documental e pesquisas bibliográficas, realizado em quatro etapas, identificação do problema, buscar de literatura, avaliação e análise de dados, e por final a síntese da revisão bibliográfica. (Oliveira, 2019)

A presente pesquisa qualitativa fora realizada de forma descritiva, através de revisão de literatura nas bases de dados da Biblioteca Virtual em Saúde (BVS), Google Acadêmico e SciELO (Eletronic Library Online) (Pereira, et al., 2019)

Após, o material encontrado foi submetido a dois momentos classificatórios, sendo que no primeiro os artigos foram selecionados considerando o título, resumo e data de publicação. Posteriormente, esses foram classificados de acordo com a sua relevância ao tema investigado, após leitura minuciosa.

Insta salientar que houve maior facilidade e interesse em busca de artigos junto ao Google Acadêmico, vez que em tal plataforma há um compilado maior de informações, pois engloba as demais plataformas anteriormente mencionadas. 
Assim, superado o momento classificatório, os artigos selecionados foram separados de modo que a organização do presente estudo se desse de uma maneira sistemática, sendo encontrados 22 artigos que contribuíram de maneira valorosa para a confecção do presente trabalho.

Assim, por meio de uma busca de dados na área da saúde em ênfase na vertente temática, como o serviço hospitalar em urgência, enfermagem em emergência, perfil de saúde, demandas ao serviço de saúde e a avaliação e análise de dados através da leitura detalhada do conteúdo e dos materiais selecionado para a síntese dos dados sumariza as informações obtidas para este estudo.

\section{Resultados e Discussão}

Este estudo analisa a importância do diagnóstico de enfermagem em urgência e emergência, sendo considerado um grande desafio, pois a cada dia, nessa área de saúde, há superlotação. Assim existem diversas barreiras para que a qualidade de atendimento e assistência médica seja mantida.

Existe uma vasta gama de material sobre o tema e não há uma divergência de entendimento sobre a urgência e emergência, e sim uma critica pela unanimidade dos autores estudos em relação ao ambiente de trabalho e os desafios advindos dessa área da saúde, pois a um trato direto com o paciente com potencial risco de vida e, também, com aquele que muitas vezes não concorda com o tempo de espera proposto pelo protocolo de Manchester. (Garcia, 2019)

Segundo o Ministério de Saúde do Brasil, o atendimento de urgência e emergência tem se caraterizado muito elevado devido as causas externas como a violência e acidentes de trânsitos, doenças crônicas, com tudo isso é necessária uma atenção maior na reorganização dos serviços de urgência e emergência para garantir uma assistência segura e de qualidade, esses atendimentos imediatos são muito importantes, mas é necessário complementar ações de promoção, prevenção e reabilitação do paciente (Brasil, 2013).

Segundo Souza (2013), devido alto crescimento no atendimento emergencial na área da saúde no Brasil, o país enfrenta múltiplos problemas como a superlotação das unidades, dimensionamento de pessoal incapacitado, rotatividade de pacientes, processo de trabalho fragmentado, conflitos de poder, no entanto mesmo com todos esses desafios a prioridade de prestar uma assistência contínua e de qualidade aos pacientes precisa de um apoio do Estado com relação aos processos de trabalho bem definidos e profissionais capacitados.

A atuação do profissional de enfermagem juntamente com a equipe multidisciplinar na sistematização de assistência de enfermagem é fundamental, sendo considerado um profissional muito importante no gerenciamento do cuidado em meio as condições adversas que aparece no cenário dos serviços de urgência e emergências, apesar das dificuldades o profissional atua sempre para garantir uma assistência com qualidade e segurança ao paciente (Felix, Rodrigues e Oliveira, 2009, Miranda et.al, 2012).

A área de atendimento de urgência e emergência, trata-se de é um setor em que não é possível a realização de uma programação prévia de atendimento, vez que no decorrer do dia podem acontecer várias situações estressantes como profissionais com dificuldades no atendimento ao paciente, falta de padronização no processo de enfermagem prático e objetivo, falta de diagnóstico correto (Maria, Quadros \& Grassi, 2012).

A resolução $n^{\circ}$. 358/2009 do COFEN relata sobre a implantação do processo de enfermagem em unidades de saúde pública e privada, responsável pelos cuidados de enfermagem como as etapas do diagnóstico e a prescrição das intervenções de enfermagem são muito importante, o que contribui totalmente para o gerenciamento no cuidado com registro sistemático e padronização dos prontuários, assim viabilizando a rotatividade e agilidade na resolução de problemas e também na valorização da atuação do profissional de saúde, contudo garantir uma assistência de qualidade ao paciente (Castro \& Caixeta, 2012). 
Por conseguinte, entende-se pela necessidade de continuidade de estudos na área, vez que se trata de um tema de alta complexidade, pois é responsável pelo pronto atendimento à pacientes portadores de várias enfermidades ou que são encaminhados ao atendimento médico devido à traumas e afins. Assim, para que o atendimento ocorra da melhor maneira possível, indica-se o estudo das emoções dos enfermeiros que atuam em tal ramo da saúde, a fim de que haja uma análise comportamental do estresse e demais emoções causadas pelo labor.

\section{Considerações Finais}

Após a pesquisa realizada para a elaboração do presente artigo, conclui-se que o diagnóstico de enfermagem em urgência e emergência é fundamental e que atribui ao profissional de enfermagem de forma objetiva as informações necessárias para a avalição do paciente, priorizando as necessidades, logo em seguida atuar na prevenção, reabilitação e promoção de saúde aos pacientes.

Em resumo o diagnóstico de enfermagem é fundamentado na investigação e documentação das informações sobre o paciente e suas necessidades, sendo elas físicas, psicológica e emocional, tendo como objetivo buscar essas informações que possam promover cuidados, intervenções inerentes ao paciente.

O diagnóstico de enfermagem na urgência e emergência é compreendido em cinco etapas, sendo elas coletas de dados, diagnostico, planejamento, implementação e avaliação, o que proporciona ao profissional de saúde um pensamento crítico, tomada de decisões, autonomia profissional e qualidade na assistência à saúde.

A atuação do profissional de saúde no local de urgência e emergência é muito importante, pois pode assegurar a segurança, eficiência, brevidade, e o livramento de risco ao paciente, assim é sem dúvida essencial a capacitação e o desenvolvimento do profissional, para desempenhar suas funções com ética e excelência.

Assim, considerando a importância do tema abordado no contexto médico, indica-se a continuidade de estudos sobre sua relevância, métodos de aprimoramento e impactos na saúde mental dos profissionais da saúde que são submetidos diariamente ao estresse causado pelo atendimento de urgência e emergência.

\section{Referências}

Brasil (2014). Consolidação das normas das redes de atenção à saúde. Ministério da Saúde. Portaria de Consolidação Nº 3 GM/MS de 28 de setembro de 2014.

Brasil (2013). Institui ações para a segurança do paciente em serviços de saúde e dá outras providências. Ministério da Saúde. ANVISA. Resolução - RDC n ${ }^{\circ}$ 36 , de 25 de julho de 2013 .

Dutra, C. S. K., Silveira, L. M., Santos, A. O., Pereira, R. \& Stabile, A. M. (2013) Diagnósticos de enfermagem prevalentes no paciente internado com sepse no Centro de Terapia Intensiva. Cogitare Enferm, 19(4):747-54

Castro, D. A. L. \& Caixeta, J.A. (2012) Sistematização da assistência de enfermagem: a importância no processo de implantação nos hospitais do brasil. Conferência internacional de estratégia em gestão, educação e sistemas de informação (CEGESI).

Conselho Federal de Enfermagem (2009). Resolução $n^{\circ}$ 358, de 15 de outubro de 2009. Dispõe sobre a sistematização da assistência de enfermagem e a implementação do processo de enfermagem em ambientes, públicos ou privados, em que ocorre o cuidado profissional de enfermagem. COFEN.

Conselho Federal de Enfermagem (2012). Resolução n. $^{\circ}$ 358, de 15 de outubro de 2012. Dispõe sobre a sistematização da assistência de enfermagem e a implementação do processo de enfermagem em ambientes, públicos ou privados, em que ocorre o cuidado profissional de enfermagem. COFEN.

Costa, E. F., Oliveira, A. D., Ferreira, I. M, Girão, K. L., \& Lopes, G. S. (2020). Aulas práticas em urgência e emergência na formação do acadêmico de enfermagem - relatos de experiência. Research, society and Development. 9(12). http://dx.doi.org/10.33448/rsd-v9i12.10411

Felix, N. N., Rodrigues, C. D. S. \& Oliveira, V. D. C. Desafios encontrados na realização da sistematização da assistência de enfermagem (SAE) em unidade de pronto atendimento. Arq. Ciência Saúde, 16 (4): 155-60.

Filho, L. A. M., Martini, J. G., Vargas, M. A. O., Reibnitz, K. S., Biterncourt, J. V. O. V. \& Lazzari, D. (2016). Competência legal do enfermeiro na urgência e emergência. Enfermagem em Foco, 7(1): 18-23.

Garcia, T. R. \& Nóbrega, M. M. L. (2009) Processo de enfermagem: da teoria à prática assistencial e de pesquisa. Esc. Anna Nery rev. Enfermagem, 13(1): $188-193$. 
Research, Society and Development, v. 10, n. 14, e159101421937, 2021

(CC BY 4.0) | ISSN 2525-3409 | DOI: http://dx.doi.org/10.33448/rsd-v10i14.21937

Maria, M. A., Quadros, F. A. A. \& Grassi, M. F. O. (2012) Sistematização da assistência de enfermagem em serviços de urgência e emergência: viabilidade de implantação. Revista Brasileira de Enfermagem, 65(2): 297-303.

Miranda, C. A., Silveira, E. N., Araújo, R. A. \& Enders, B. C. (2012). Opinião de enfermeiros sobre instrumentos de atendimento sistematizado a paciente em emergência. Revista Rene, 13(2): 396-407.

Munhoz, O. L., Andolhe, R, Magnano, T. S. B. S., Mendes, T. C. \& Guedes, R. (2016). Atuação do enfermeiro em unidade de pronto socorro: relato de experiência. Biblioteca Lascasas, 12(1).

North American Nursing Association. (2009-2011). Diagnósticos de enfermagem da NANDA: definições e classificação. Artmed

Oliveira, N. O, Ramos, B. J., Piazza, M., Prado, M. L., Reibnitz, K. S. \& Souza, A. C. (2015). Unidade de pronto atendimento-UPA 24H: Percepção da enfermagem. Texto contexto enfermagem, 24(1): 238-244.

Passos, S. S. S. \& Sadigusky, D. (2011). Cuidado de Enfermagem ao paciente dependente e hospitalizado. Rev. Enferm, 19(4):598-603.

Penedo, R. M. \& Spin, W. C. (2014). Significado da sistematização da assistência de enfermagem para enfermeiros gerentes. Acta Paul Enfermagem, (2791): $86-92$.

Rocha, E. C. A. (2012). Atuação da enfermagem em urgência e emergência. Revista Unitins, 3(1): 1- 12.

Sallum, A. M. C., Santos, J. L. F. \& Lima, F. D. (2015). Diagnósticos de enfermagem em vítimas fatais decorrentes de trauma no cenário da emergência. Rev. Latino-Am. Enfermagem, 2(1).

Silva, D. G. \& Vargas, C. R. Sistematização da assistência de enfermagem: aspectos éticos legais e a importância na prática profissional do enfermeiro. Revista Científica da Faculdade de Educação e Meio Ambiente, 2 (1): 22-41, 2011.

Soares, M. I., Rescz, Z. M. R., Terra, F. S. \& Camelo, S. H. H. (2015) Sistematização da assistência de enfermagem: facilidades e desafios do enfermeiro na gerência da assistência. Escola Anna Nery Revista de Enfermagem, 19(1).

Souza, M. F. Cianclarullo T. I., et al (2012). As teorias de enfermagem e sua influência nos processos cuidativos. organizadores. Sistema de assistência de enfermagem: evolução e tendências. Ícone (5a ed.). 